\title{
In Vivo Evaluation of the Biomechanical Properties of Optic Nerve and Peripapillary Structures by Ultrasonic Shear Wave Elastography in Glaucoma
}

\author{
Atilla Suleyman Dikici, ${ }^{1}$ Ismail Mihmanli, ${ }^{1}$ Fahrettin Kilic, ${ }^{1}$ Ahmet Ozkok, ${ }^{2}$ Gokhan Kuyumcu, ${ }^{1}$ Pinar \\ Sultan, ${ }^{2}$ Cesur Samanci, ${ }^{1}$ Mehmet Halit Yilmaz, ${ }^{1}$ Babak Rafiee, ${ }^{1}$ Nevbahar Tamcelik, ${ }^{2}$ Zehra Isik \\ Hasiloglu, ${ }^{1, *}$ and Fatih Kantarci ${ }^{1}$ \\ ${ }^{1}$ Department of Radiology, Istanbul University Cerrahpasa Medical Faculty, Istanbul, Turkey \\ ${ }^{2}$ Department of Ophthalmology, Istanbul University Cerrahpasa Medical Faculty, Istanbul, Turkey \\ "Corresponding author: Zehra Isik Hasiloglu, Department of Radiology, Kocamustafapasa Fatih Istanbul, Istanbul University Cerrahpasa Medical Faculty, Istanbul, Turkey. Tel: \\ +90-5336278557; Fax: 90-2124143167, E-mail: zhasiloglu@gmail.com
}

Received 2016 February 03; Revised 2016 February 27; Accepted 2016 March 15.

\begin{abstract}
Background: Primary open-angle glaucoma is a multifactorial serious disease characterized by progressive retinal ganglion cell death and loss of visual field.

Objectives: The purposes of this study were to investigate shear wave elastography (SWE) use in the evaluation of the optic nerve $(\mathrm{ON})$ and peripapillary structures, and to compare the findings between glaucomatous and control eyes.

Patients and Methods: A case-controlled study, including 21 patients with primary open-angle glaucoma and 21 age-matched control subjects, was carried out. All of the participants had comprehensive ophthalmological exams that included corneal biomechanical measurements with ocular response analyzer. In vivo evaluation of the biomechanical properties of the ON and peripapillary structures were performed with SWE in all participants. The Kolmogorov-Smirnov test was used to analyze the normal distribution of data. Differences of parameters in ophthalmologic data and stiffness values of patients with and without glaucoma were evaluated using the Mann-Whitney U test.

Results: There were no statistically significant differences between the glaucoma and control groups in terms of age $(P>0.05)$ and gender $(\mathrm{P}>0.05)$. Corneal hysteresis was lower in the glaucoma group $(\mathrm{P}<0.05)$. Corneal compensated intraocular pressure and Goldmann correlated intraocular pressure were higher in the glaucoma group ( $\mathrm{P}<0.0001$ for both). The mean stiffness of the ON and peripapillary structures were significantly higher in glaucoma patients for each measured region $(\mathrm{P}<0.05)$.

Conclusion: The study evaluated the biomechanical properties of the ON and peripapillary structures in vivo with SWE in glaucoma. We observed stiffer ON and peripapillary tissue in glaucomatous eyes, indicating that SWE claims new perspectives in the evaluation of ON and peripapillary structures in glaucoma disease.
\end{abstract}

Keywords: Glaucoma, Optic Nerve, Shear Wave Elastography, Peripapillary Structures

\section{Background}

Glaucoma affects more than 60 million people worldwide and it is the most common cause of irreversible vision loss (1). It is a term describing a group of ocular disorders with multifactorial etiology united by a clinically characteristic intraocular pressure-associated optic neuropathy (2). The optic nerve $(\mathrm{ON})$ is mainly constituted of retinal ganglion cell (RGC) axons and these axons leave the eye by passing through the lamina cribrosa (LC). The LC and peripapillary sclera (ppSC) have a barrier function between the intraocular and retrobulbar spaces (3). As the pressure in the intraocular space is higher than the retrobulbar space, biomechanical resistance of the LC is also important for both anatomical and functional stability of the ON. Increased cupping of the ON head is a finding of glaucoma
(Figure 1). The LC region of the $\mathrm{ON}$ is thought to be the primary location of the RGC axonal insult in glaucoma (4).

Evaluation of the biomechanical properties of ocular tissues has the potential to provide invaluable information that aids in the diagnosis and management of many eye diseases, including glaucoma (5). The ocular response analyzer (ORA, Reichert Ophthalmic Instruments, Depew, NY) is a relatively new device capable of measuring the biomechanical properties of the cornea (6). The ORA measures four main parameters: corneal hysteresis $(\mathrm{CH})$, corneal resistance factor (CRF), Goldmann correlated intraocular pressure $\left(\mathrm{IOP}_{\mathrm{G}}\right)$, and corneal compensated intraocular pressure $\left(\mathrm{IOP}_{\mathrm{CC}}\right)$. The ORA obeys the non-contact tonometry principles. The liberation of an accurately measured air pulse applies an air puff to the cornea. $\mathrm{CH}$ is the 

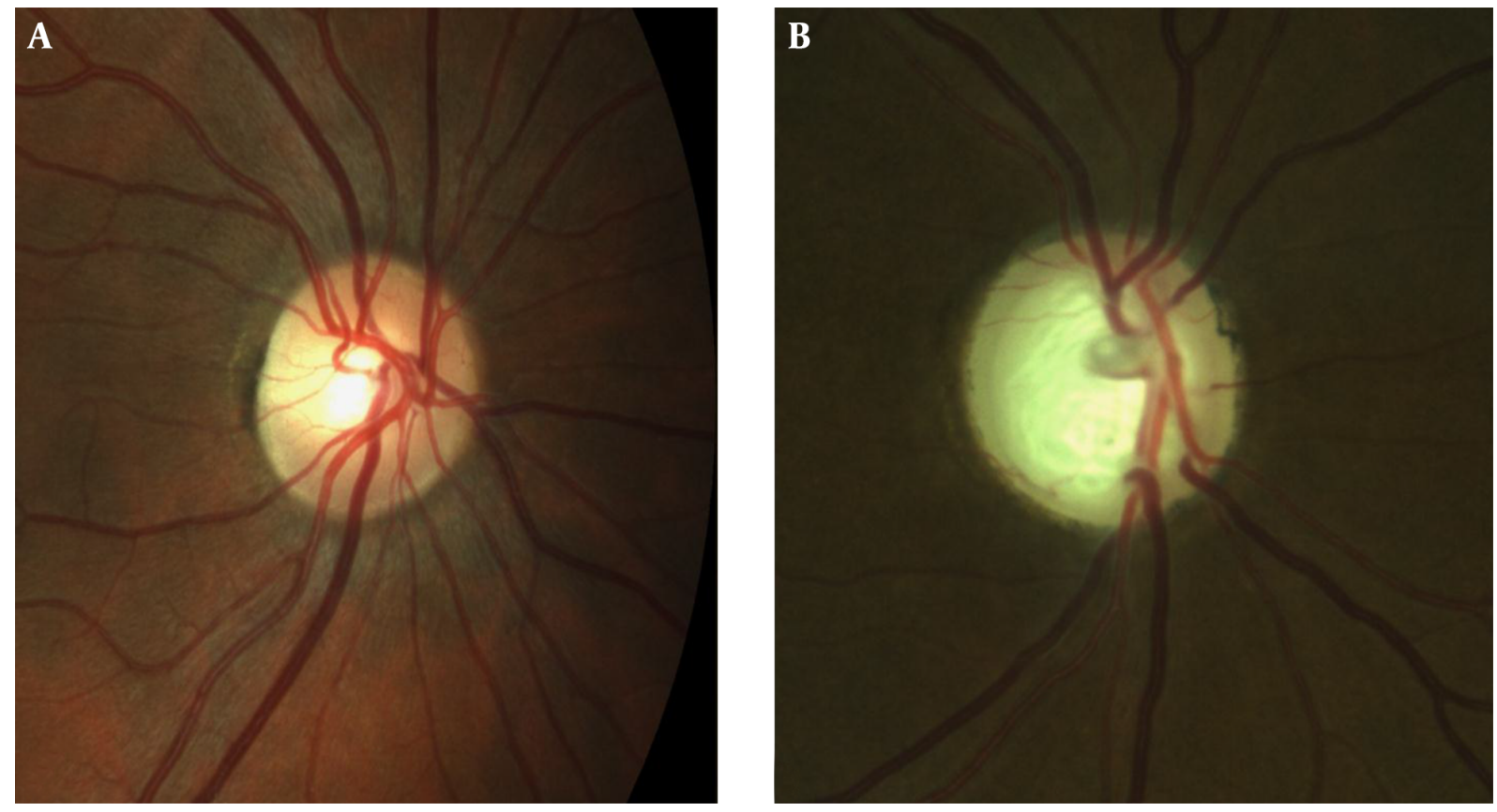

Figure 1. Optic disc appearance of normal (A) and glaucomatous (B) eyes. Advanced cupping of optic disc could be seen in glaucomatous eye.

difference between pressure values acquired during first and second applanations (P1 and P2) and it is a measure of corneal viscoelasticity. CRF is calculated using the formula P2-0.43P1 and it is an indicator of the overall resistance of the cornea. $\mathrm{IOP}_{\mathrm{CC}}$ is a measure of intraocular pressure that is free of corneal influence and $\mathrm{IOP}_{\mathrm{G}}$ is defined as the average of P1 and P2. Although the ORA is a reliable device to evaluate corneal resistance, it cannot evaluate the biomechanical properties of the other anatomical locations of the eye, especially LC, which is important for glaucoma pathogenesis.

The most important risk factor for glaucoma is high intraocular pressure (IOP) and IOP reduction is the only proven treatment for glaucoma. However, there is a wide range of individual susceptibility to IOP. Biomechanical properties of LC and PPSC have a partial role in individual susceptibility to IOP. Recent studies have contributed to a new understanding of the mechanical interactions between the LC and ppSC and how the alterations of these structures potentially affect the susceptibility of RGC axons to glaucomatous damage (7-10). Structural alterations in the collagen and elastic fibers of the LC and PpSC have been reported in transmission electron microscopy studies in post-mortem human glaucomatous eyes (11). Biomechanical changes in the LC and pPSC have also been shown in glaucomatous eyes (12). Therefore, the importance of alterations in the biomechanical properties of the LC and
ppSC is known in glaucoma pathogenesis. Unfortunately, there is no direct in vivo method to evaluate the biomechanical properties of the LC, PpSC, and ON.

Elastography is a new, non-invasive ultrasonographic imaging technique providing the structural stiffness data to understand material properties indirectly as an addition to conventional grayscale ultrasonography findings. There are four types of elastography techniques: strain, transient, shear wave elastography, and acoustic radiation force impulse imaging. The new generation realtime quantitative elasticity imaging technique, shear wave elastography (SWE), has been recently introduced and successful studies concerning breast, thyroid, liver, and musculoskeletal system have been reported (13-18). SWE has a different application philosophy regarding strain elastography. This technique does not use compressiondecompression effects to obtain stiffness information. It delivers shear waves to represent the quantitative stiffness values in kilopascals ( $\mathrm{kPa}$ ). The theoretical advantage of SWE is that it provides compression-free information on shear wave speed as acoustic radiation force impulses (ARFI) do in a 2-D area of varying centimeters, but not in point or line as do ARFI or transient elastography. SWE applies radiation force impulses focused at various depths while ARFI's radiation force focuses at a constant depth. 


\section{Objectives}

The aims of the current study are to determine the potential role of SWE in evaluating the elasticity properties of the ON and ppSC and to compare these parameters between healthy subjects and glaucoma patients. To the best of our knowledge, the use of SWE for the biomechanical properties of the PpSC and ON has not been studied previously.

\section{Patients and Methods}

This study was conducted in the ophthalmology and radiology departments of Istanbul University, Cerrahpasa Medical Faculty between March 2013 and March 2014. The study included 42 eyes of 21 consecutive primary openangle glaucoma patients and 42 eyes of 21 healthy volunteers, for a total of 84 eyes. Written informed consent was obtained from all patients and healthy volunteers. The study was approved by the local ethics committee of Istanbul University and was designed in accordance with the declaration of Helsinki.

The control group consisted of age- and sex-matched healthy volunteers from hospital employees. Study and control groups underwent ophthalmological evaluation; measurements were taken by experienced ophthalmologists (NT, AO) in the Ophthalmology Department. An experienced radiologist (FK, six years of experience in the US, three years of experience with elastography) who was blind to the clinical information performed sonographic and elastographic evaluations. The patients were under the control of medication therapy; ten of them (47.6\%) were less than three years, eight of them (38.1\%) between three to five years, and three of them (14.3\%) were more than 5 years. The patients who had any ocular disease or who underwent ocular surgery were not included in the study.

\subsection{Ophthalmological Evaluation}

Consecutive volunteers with a diagnosis of refractive error less than 3.00 diopters constituted the control group and patients with glaucoma formed the glaucoma group. All subjects underwent a comprehensive ophthalmological examination, including Goldmann Applanation Tonometry (GAT) and fundoscopy. Humphrey visual field tests, which had been done within the last three months before SWE measurements, mean deviation (MD) and pattern standard deviation (PSD) parameters, and cup-to-disc ratios (C/D) were obtained from the medical charts of subjects with glaucoma. MD indicates the total amount of visual field loss and PSD indicates the focality of visual field loss (19). The unit of measure for both MD and PSD is the decibel (dB). An enhanced glaucoma staging system (eGSS) was used to determine the stage of glaucoma (20). Central corneal thickness (CCT) was measured with an ultrasound pachymeter (US 4000, Nidek, Japan). An ocular response analyzer (ORA, software 2.04, Reichert Ophthalmic instruments, Depew, NY) was used to measure $\mathrm{CH}, \mathrm{CRF}, \mathrm{IOP}_{\mathrm{CC}}$, and $\mathrm{IOP}_{\mathrm{G}}$.

\subsection{Radiological Evaluation}

After ophthalmologic examination, patients underwent radiological evaluation on the same day. All examinations were performed with the Aixplorer US system (SuperSonic Imagine, Aix-en-Provence, France) equipped with a 4-15 MHz linear transducer. Sonographic measurements were taken in a supine position. The patients were told to look at a constant marker on the ceiling while the eyelids were closed and not to move their eyes. A probe was placed on the upper lids of closed eyes in the axial plane of the orbit and an angulating probe was placed about $15-30^{\circ}$ caudally to better visualize the ON. In grayscale sonography, eyes were scanned for any possible morphologic pathology due to intraocular diseases and masses. The anteriorposterior diameter of the vitreous chamber, transducer to fovea distance, and mediolateral diameter of the intraorbital ON just adjacent to the intraocular part were measured (Figure 2).

After grayscale examination, the SWE was performed. Ten healthy control subjects were selected for the purpose of standardization of the elastographic technique. These subjects were not included in the study and statistical analyses. During SWE, aiming to avoid any compression effect of the transducer, the operator placed the transducer onto the eyelid with light contact using excessive gel and kept the transducer stationary during acquisitions. If possible, a gap between the probe and eyelid was maintained. The patients were instructed not to move their eyes to obtain accurate measurements.

The machine software displayed elastograms as an overlay in dual mode (vertical/horizontal) simultaneously with grayscale images. The sonography window was adjusted to the region of interest. An adjustable rectangular electronic box, which was automatically provided by the system software, was placed on the elastogram screen. The electronic box displayed the tissue stiffness in real-time using a chromatic scale (spectrum from blue to red) indicating low to high shear modulus (stiffness). The integrated SWE software allowed for placement of circular regions of interest (ROIs) of various diameters within the elastography window and automatically displayed shear modulus data (in kilopascals, $\mathrm{kPa}$ ) for the ROIs. The sizes of the ROIs were fixed to $2 \mathrm{~mm}$ in all cases. The ROIs were placed on four different parts of the eye: the nasal and temporal sides 


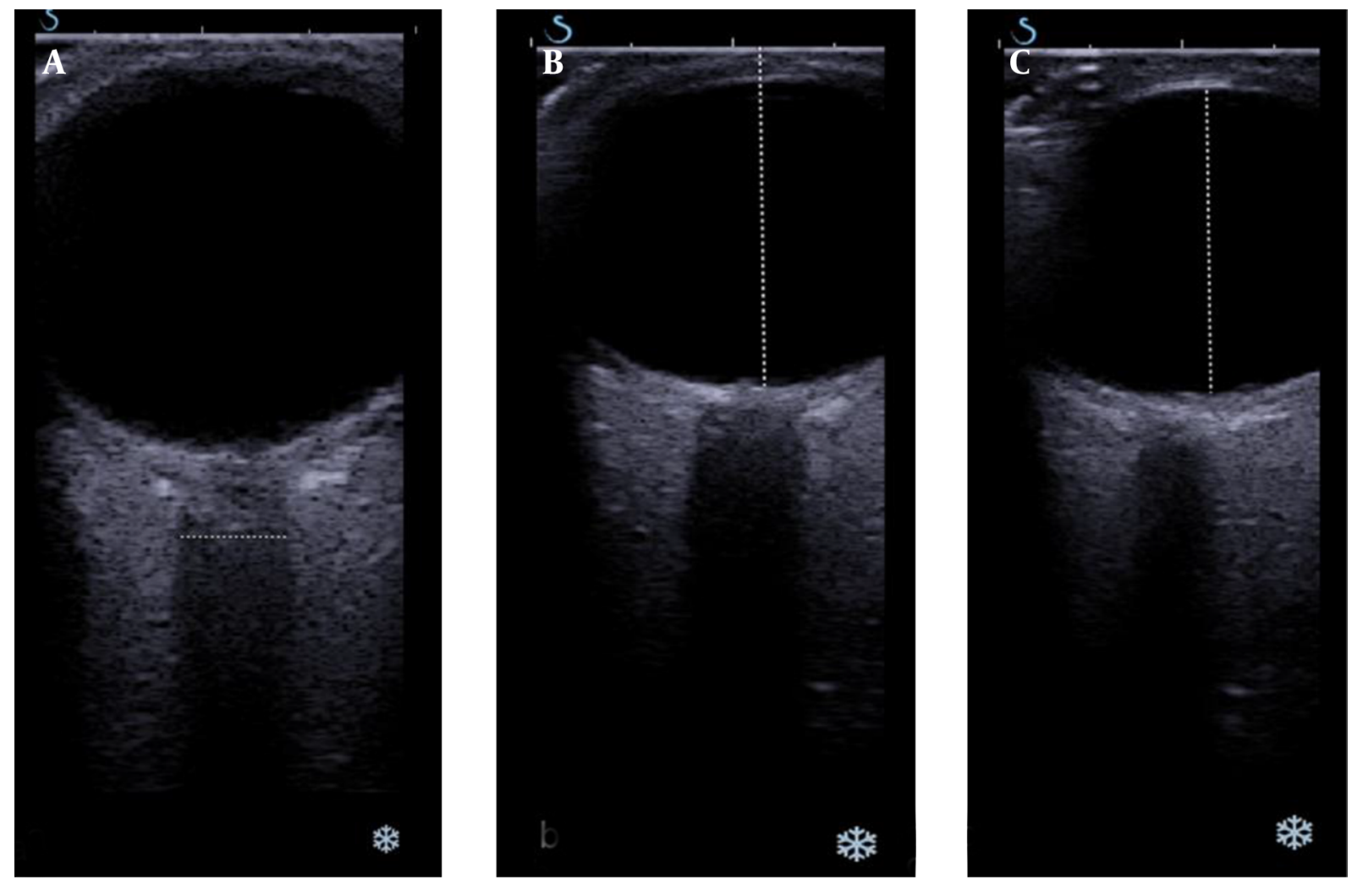

Figure 2. Grayscale sonography evaluation of the orbit. A, Measurement of optic nerve thickness just adjacent to the orbit; B, In the same region, measurement of transducer to fovea distance and C, Anterior-posterior diameter of the vitreous chamber.

of the perineural sclera and the distal and globe conjugating parts of the ON. The mean stiffness values and standard deviation data within each ROI were obtained. Care was taken not to place the circular ROIs outside the $\mathrm{ON}$ and sclera (Figure 3).

\subsection{Statistical Analysis}

Statistical analyses were performed using SPSS software (version 16.0, SPSS Inc., Chicago, IL, USA). The maximum and mean stiffness within the ROIs were expressed as the mean of three different measurements. The Kolmogorov-Smirnov test was used to analyze the normal distribution of data. Differences in age, $\mathrm{IOP}_{\mathrm{CC}}, \mathrm{IOP}_{\mathrm{G}}, \mathrm{CH}, \mathrm{CRF}$, cup to disc (C/D) ratio, vitreous diameter, orbit's diameter, ON diameter, and shear elastic modulus data of the perineural sclera and ON of patients with and without glaucoma were evaluated using the Mann-Whitney U test. All measurements are expressed as mean \pm two standard deviations. All of the SWE data was correlated to ophthalmologic measures using a Spearman Correlation test. A P value $<0.05$ was considered to be statistically significant.

\section{Results}

Table 1 represents the mean patient age and the grayscale sonographic and ocular findings of patients diagnosed with glaucoma and healthy controls. All of the patients were Caucasian. There were no statistically significant differences between the two groups in terms of age $(P=0.07)$ and gender $(P=0.711)$. All ORA parameters were significantly different between the glaucoma and control groups except for CRF. CH was lower in the glaucoma group $(9.7 \pm 1.5)$ compared to the control group $(10.9 \pm 1.3)(\mathrm{P}=$ $0.002)$. $\mathrm{IOP}_{\mathrm{CC}}$ and $\mathrm{IOP}_{\mathrm{G}}$ were higher in the glaucoma group compared to the control group ( $\mathrm{P}<0.0001$ for both), confirming the disease. The mean MD and PSD values were (-5.8 $\pm 8.2)$ and (5.0 \pm 4.5$)$, respectively. The mean C/D ratio was $0.53 \pm 0.23$ in the glaucoma group. Of the 42 eyes in the glaucoma group, 24 (57\%) had stage I, 8 (19\%) had stage II, 3 (7\%) had stage III, 4 (10\%) had stage IV, and 3 (7\%) had stage V glaucoma according to eGSS classification.

Grayscale sonography of the orbit allows for morphological evaluation. Globe and vitreous axial diameters were not significantly different in the two groups. ON 


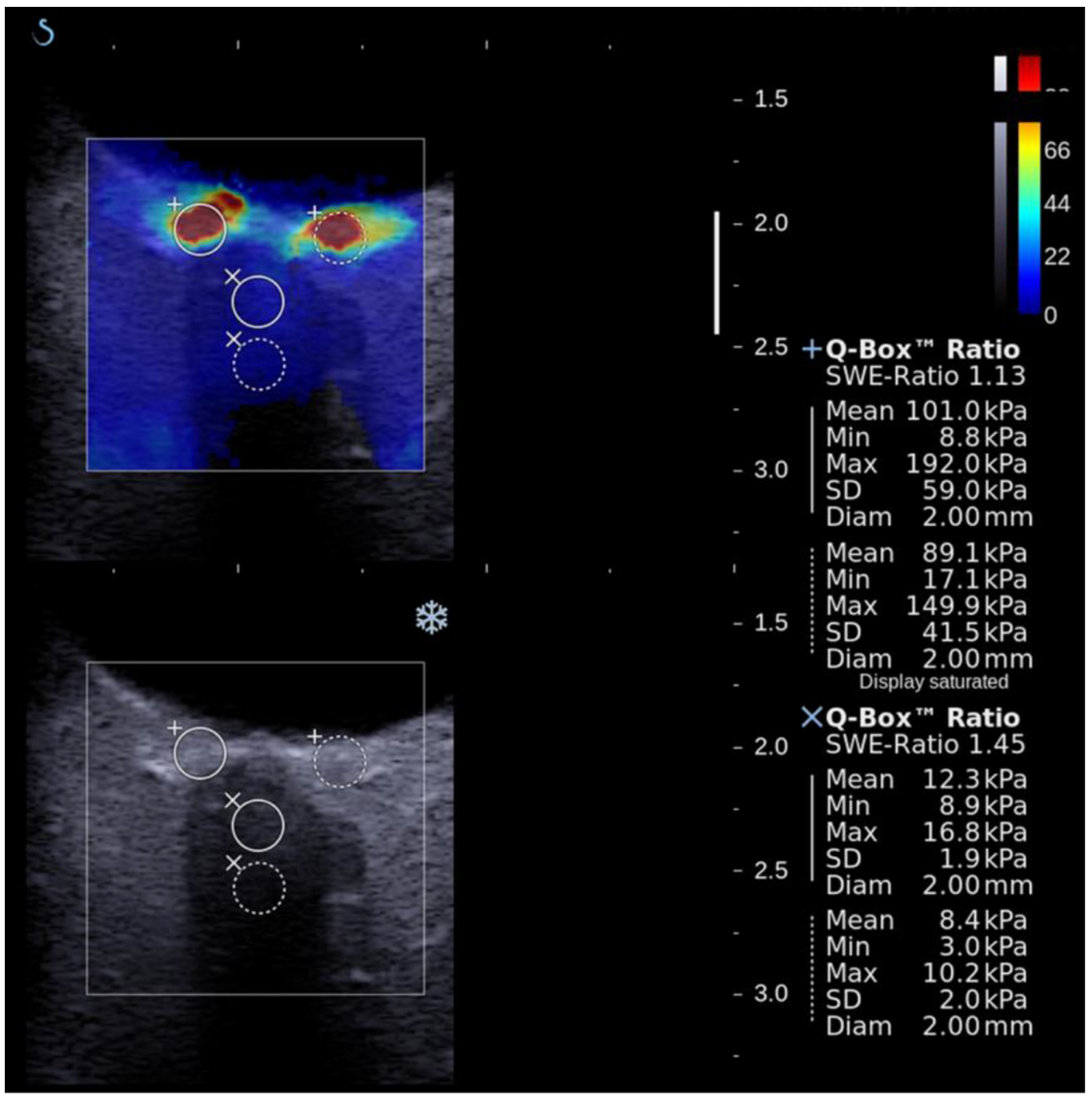

Figure 3. Shear wave elastography evaluation of the optic nerve and perineural sclera. Measurements were taken from nasal and temporal sides of perineural sclera, distal, and globe conjugating part of the optic nerve by the help of ROI placement.

diameters were significantly different between the two groups; thinner ONs were found in glaucomatous eyes (3.54 \pm 0.83 vs. $3.07 \pm 0.73 \mathrm{~mm}$, respectively, $\mathrm{P}=0.009$ ) In addition, the $\mathrm{ON}$ diameter was inversely correlated with the $\mathrm{C} / \mathrm{D}$ ratio (Pearson $\mathrm{r}=-257, \mathrm{P}=0.009$ ).

The mean stiffness values were obtained from both horizontal sides of the ppSC and intraorbital parts of the ON. The mean stiffness values were significantly higher in glaucoma patients for each measured region $(\mathrm{P}<0.05$; Table 2). While investigating the correlations of these differences, the important points were noted. Increased stiffness values of both nasal and temporal perineural scleral and intraorbital distal parts of ON were inversely correlated with the ON diameter (Pearson $r=-0.259,-0.274$, and -0.200 , respectively). In addition to the significant correlation of ON diameter with the C/D ratio, the mean stiffness 
Table 1. The Grayscale Sonography and Oculometric Findings of Patients Diagnosed With Glaucoma and Normal Controls

\begin{tabular}{lccc}
\hline & Glaucoma & Control & PValue \\
\hline Patient's Age, $\mathbf{y}$ & $61.29 \pm 9.76$ & $60.10 \pm 10.2$ & 0.07 \\
\hline Globe AP diameter, $\mathbf{~ m m}$ & $22.29 \pm 1.42$ & $22.43 \pm 1.30$ & 0.496 \\
\hline Vitreous AP diameter, $\mathbf{m m}$ & $18.84 \pm 0.93$ & $18.97 \pm 0.98$ & 0.607 \\
\hline Optic nerve diameter, $\mathbf{m m}$ & $3.07 \pm 0.73$ & $3.51 \pm 0.83$ & 0.009 \\
\hline IOP $_{\text {CC }}$ & $19.76 \pm 3.37$ & $14.91 \pm 2.90$ & $<0.001$ \\
\hline IOP $_{\text {G }}$ & $19.01 \pm 3.45$ & $14.89 \pm 3.28$ & $<0.001$ \\
\hline CH & $9.74 \pm 1.53$ & $10.87 \pm 1.31$ & 0.002 \\
\hline CRF & $10.93 \pm 1.68$ & $10.74 \pm 1.61$ & 0.619 \\
\hline C/D Ratio & $0.53 \pm 0.23$ & $0.23 \pm 0.04$ & 0.000 \\
\hline
\end{tabular}

Abbreviations: AP; anteroposterior, C/D; cup to disc, CH, corneal hysteresis; CRF, corneal resistance factor; IOPCC, corneal compensated intraocular pressure; IOPG, Goldmann correlated intraocular pressure.

values of the temporal and nasal parts of the sclera and the distal part of the $\mathrm{ON}$ were correlated with the C/D ratio (Pearson $r=0.310,0.319$, and 0.265 , respectively). There was no correlation between visual field parameters and elastography parameters.

Table 2. Shear Wave Elastography Findings of Patients Diagnosed With Glaucoma and Normal Controls

\begin{tabular}{lccc}
\hline & Glaucoma & Control & PValue \\
\hline $\begin{array}{l}\text { Optic nerve distal mean } \\
\text { elastic modulus, kPa }\end{array}$ & $22.03 \pm 17.8$ & $17.3 \pm 13.3$ & 0.005 \\
\hline $\begin{array}{l}\text { Optic nerve distal end } \\
\text { mean elastic modulus, kPa }\end{array}$ & $19.36 \pm 14.28$ & $12.6 \pm 8.9$ & 0.005 \\
\hline $\begin{array}{l}\text { Perineural sclera, nasal } \\
\text { mean elastic modulus, kPa }\end{array}$ & $89.01 \pm 44.33$ & $66.30 \pm 24.06$ & 0.006 \\
$\begin{array}{l}\text { Perineural sclera, temporal } \\
\text { mean elastic modulus, kPa }\end{array}$ & $71.80 \pm 39.28$ & $52.6 \pm 28.30$ & 0.017 \\
\hline
\end{tabular}

\section{Discussion}

In this study, we demonstrated a novel use of SWE technology to evaluate the biomechanical properties of the ppSC and ON and studied whether these elasticity measurements correlated with any clinical variables in glaucoma patients. As is known, there is currently no available in vivo method to evaluate the biomechanical properties of the ppSC and ON. Evaluations in the study showed that the mean stiffness values were significantly higher in the perineural sclera's nasal and temporal regions and the ON in glaucomatous eyes compared to healthy controls ( $\mathrm{P}<$ 0.05).
Chronic high intraocular pressure may increase the structural stiffness of the ppSC and directly lead to LC deformation (21). It is well known that the deformative changes of the ppSC develop under the strain and stress of the disease course and may have potential roles in glaucoma pathogenesis. Chronic intolerably high IOP may cause biomechanical changes in the ppSC and it may affect the susceptibility of ganglion cell axons. Hence, it is important to show ppSC changes when attempting to understand the effect of IOP on the ON head.

Changes that occur in the $\mathrm{ON}$ are the most prominent features of glaucomatous damage. Studies on the morphology of the ON head in monkeys $(22,23)$ and ON head biomechanics in humans $(8,24)$ have increased our knowledge about the biomechanics of the ON head and peripapillary structures in glaucoma. These studies all underline the significant deformative/degenerative changes in the material properties and architecture of the ppSC as well as the $\mathrm{ON}$, hence aiding the understanding of the impact of glaucomatous disease. Higher stiffness values among glaucoma patients in the current study may be a reflection of direct/indirect effects of the intolerably high IOP on the target tissues. Higher elasticity may also be a predisposing risk factor in glaucoma development and vice versa. However, we showed no histopathological correlation with SWE findings.

The stiffness values were correlated inversely with the mean thickness of the ON, which was lower in glaucomatous patients $(3 \mathrm{~mm})$ than in normal eyes $(3.5 \mathrm{~mm}$; $\mathrm{P}<$ 0.05). Furthermore, the $C / D$ ratio, which is an important ophthalmoscopy finding, was well correlated with stiffness values. We did not find a correlation between visual field (i.e., MD and PSD) and elastography parameters. We have two possible explanations for this finding. First, our sample size was not large and most of the glaucoma patients included in the study had mild to moderate glaucoma. Therefore, their functional damage may be associated with less anatomic and morphologic damage. Visual field parameters reflect the functional status of the $\mathrm{ON}$ and functional damage has been reported to be affected relatively late, compared to anatomical changes, in the course of glaucoma. Second, peripapillary stiffness increases in glaucomatous patients may be a preliminary indication of glaucoma rather than a result of glaucomatous damage. As a potential prognostic factor, stiffness increases might be discovered in the early stages of the disease.

Herein, we investigated the usefulness of SWE in in vivo evaluation of the ON and peripapillary structures in glaucomatous eyes and healthy control eyes. There were significant differences in the stiffness values of the $\mathrm{ON}$ and peripapillary structures in glaucomatous and healthy eyes with SWE. Also, our results obtained from SWE were cor- 
related with the C/D ratio, which indicates the severity of glaucoma and IOP, which is the main parameter of follow up for ophthalmologists. The correlations between the C/D ratio, ON diameter, and higher SWE measures suggested that SWE has the potential to aid in the diagnosis of glaucoma. Interestingly, beyond a glaucomatous impact, SWE did not indicate a high correlation with IOP increases directly. The results were correlated with the degenerative changes in the ON and ppSC.

The optic nerve color coding in SWE imaging is a bit slower than the peripapillary sclera. Gennison et al. published a study on SWE imaging that investigates the effect of muscle fiber orientation on SWE imaging $(8,24)$. A significant difference of SWE values was revealed in different orientations of probe, parallel or perpendicular, on the muscle. However, there was no way to image the optic nerve in parallel orientation with ultrasonography probe in our study. In all cases, we obtained SWE values of the optic nerve regardless of the patient characteristics. Hence, the study depicts the glaucoma effect on the optic nerve and does not intend to suggest a diagnostic threshold level.

In a recent study, Agladioglu et al. investigated the elasticity index and strain ratio of orbital and retro-orbital structures in glaucomatous patients by strain elastography imaging (25). According to this study, there were no statistical differences in the strain elasticity index of these structures between glaucomatous patients and healthy controls. For instance, there was a statistical difference in the strain ratio of anterior and posterior vitreous among glaucomatous eyes and the healthy controls. First, semi-quantitative methodology of the strain elastography might be the cause of insignificant results. Secondly, investigating such a small area with the strain elastography is subjective regarding SWE, which can evaluate the $2 \mathrm{~mm}$ thin areas quantitatively by small ROI calibrating. Furthermore, fluid content of the vitreous might have an insufficient inductive role of mechanical compression due to the elastic nature of vitreous.

Although considerable differences were noted between glaucomatous and normal eyes in general, an overlap of stiffness between patients with glaucoma and healthy controls was noticed. This may be due to other factors playing a role in the pathogenesis of glaucoma. Two main limitations of the current study include a small sample size and relatively small number of patients with advanced glaucoma. As this is the first in vivo study, further studies with a larger patient sample size are needed.

Initial findings suggest that the method has potential use in the disease. The representation of glaucomatous damage should deliver new insights for patient evaluation. Patients with restricted ophthalmologic evaluation due to red eye, leucoria, and cataract disease are potential sub- jects of the SWE imaging. Long-term evaluation of glaucoma damage to optic nerve could be accomplished before field of view injury develops.

In conclusion, this manuscript discusses evaluating the biomechanical properties of peripapillary tissue in glaucoma in vivo. The study compared elasticity parameters in healthy subjects and glaucomatous patients. We found that the SWE technique is potentially a valuable tool for evaluation of the peripapillary region, particularly in glaucoma. We observed stiffer ppSC and ON SWE values in eyes with glaucoma and the elastography values were correlated with anatomical parameters. Further studies on how best to use SWE for the diagnosis and risk evaluation of glaucoma and other optic neuropathy diseases are needed.

\section{Acknowledgments}

Istanbul university Cerrahpasa medical school, radiology and ophthalmology departments' workers.

\section{Footnotes}

Authors' Contribution: Study concept and design: Atilla Suleyman Dikici, Zehra Isik Hasiloglu, Fahrettin Kilic, Fatih Kantarci, Ahmet Ozkok, Pinar Sultan; acquisition of data: Atilla Suleyman Dikici, Fahrettin Kilic, Cesur Samanci, Gokhan Kuyumcu, Nevbahar Tamcelik; analysis and interpretation of data: Fahrettin Kilic, Cesur Samanci, Gokhan Kuyumcu, Pinar Sultan; drafting of the manuscript: Atilla Suleyman Dikici, Ismail Mihmanli, Mehmet Halit Yilmaz, Ahmet Ozkok, Babak Rafiee; critical revision of the manuscript for important intellectual content: Zehra Isik Hasiloglu, Fatih Kantarci, Ismail Mihmanli, Nevbahar Tamcelik; statistical analysis: Cesur Samanci, Fatih Kantarci; administrative, technical, and material support: Atilla Suleyman Dikici, Zehra Isik Hasiloglu, Fatih Kantarci, Mehmet Halit Yilmaz, Pinar Sultan, Babak Rafiee; study supervision: Atilla Suleyman Dikici, Zehra Isik Hasiloglu, Fatih Kantarci.

Financial Disclosure: The authors have no relevant financial interest in this article. There is no conflict of interest with any financial organization regarding the material discussed in the manuscript.

\section{References}

1. Pascolini D, Mariotti SP. Global estimates of visual impairment: 2010. Br J Ophthalmol. 2012;96(5):614-8. doi: 10.1136/bjophthalmol-2011300539. [PubMed: 22133988].

2. Casson RJ, Chidlow G, Wood JP, Crowston JG, Goldberg I. Definition of glaucoma: clinical and experimental concepts. Clin Experiment Ophthalmol. 2012;40(4):341-9. doi: 10.1111/j.1442-9071.2012.02773.x. [PubMed: 22356435]. 
3. Jonas JB, Berenshtein E, Holbach L. Anatomic relationship between lamina cribrosa, intraocular space, and cerebrospinal fluid space. Invest Ophthalmol Vis Sci. 2003;44(12):5189-95. [PubMed: 14638716].

4. Crawford Downs J, Roberts MD, Sigal IA. Glaucomatous cupping of the lamina cribrosa: a review of the evidence for active progressive remodeling as a mechanism. Exp Eye Res. 2011;93(2):133-40. doi: 10.1016/j.exer.2010.08.004. [PubMed: 20708001].

5. Ozkok A, Tamcelik N, Ozdamar A, Sarici AM, Cicik E. Corneal viscoelastic differences between pseudoexfoliative glaucoma and primary open-angle glaucoma. J Glaucoma. 2013;22(9):740-5. doi: 10.1097/IJG.0000000000000036. [PubMed: 24299728].

6. Luce DA. Determining in vivo biomechanical properties of the cornea with an ocular response analyzer. J Cataract Refract Surg. 2005;31(1):156-62. doi: 10.1016/j.jcrs.2004.10.044. [PubMed: 15721708].

7. Sander EA, Downs JC, Hart RT, Burgoyne CF, Nauman EA. A cellular solid model of the lamina cribrosa: mechanical dependence on morphology. J Biomech Eng. 2006;128(6):879-89. doi: 10.1115/1.2354199. [PubMed: 17154690].

8. Sigal IA, Flanagan JG, Ethier CR. Factors influencing optic nerve head biomechanics. Invest Ophthalmol Vis Sci. 2005;46(11):4189-99. doi: 10.1167/iovs.05-0541. [PubMed:16249498].

9. Sigal IA, Flanagan JG, Tertinegg I, Ethier CR. Predicted extension, compression and shearing of optic nerve head tissues. Exp Eye Res. 2007;85(3):312-22. doi: 10.1016/j.exer.2007.05.005. [PubMed: 17624325].

10. Braunsmann C, Hammer CM, Rheinlaender J, Kruse FE, Schaffer TE, Schlotzer-Schrehardt U. Evaluation of lamina cribrosa and peripapillary sclera stiffness in pseudoexfoliation and normal eyes by atomic force microscopy. Invest Ophthalmol Vis Sci. 2012;53(6):2960-7. doi: 10.1167/iovs.11-8409. [PubMed: 22491409].

11. Hernandez MR, Pena JD. The optic nerve head in glaucomatous optic neuropathy. Arch Ophthalmol. 1997;115(3):389-95. [PubMed: 9076213].

12. Coudrillier B, Tian J, Alexander S, Myers KM, Quigley HA, Nguyen TD. Biomechanics of the human posterior sclera: age- and glaucomarelated changes measured using inflation testing. Invest Ophthalmol Vis Sci. 2012;53(4):1714-28. doi: 10.1167/iovs.11-8009. [PubMed: 22395883].

13. Berg WA, Cosgrove DO, Dore CJ, Schafer FK, Svensson WE, Hooley RJ, et al. Shear-wave elastography improves the specificity of breast US: the BE1 multinational study of 939 masses. Radiology. 2012;262(2):435-49. doi: 10.1148/radiol.11110640. [PubMed: 22282182].

14. Cosgrove DO, Berg WA, Dore CJ, Skyba DM, Henry JP, Gay J, et al. Shear wave elastography for breast masses is highly reproducible. Eur Radiol. 2012;22(5):1023-32. doi: 10.1007/s00330-011-2340-y. [PubMed: 22210408].
15. Tanter M, Bercoff J, Athanasiou A, Deffieux T, Gennisson JL, Montaldo $\mathrm{G}$, et al. Quantitative assessment of breast lesion viscoelasticity: initial clinical results using supersonic shear imaging. Ultrasound Med Biol. 2008;34(9):1373-86. doi: 10.1016/j.ultrasmedbio.2008.02.002. [PubMed: 18395961].

16. Evans A, Whelehan P, Thomson K, McLean D, Brauer K, Purdie C, et al. Quantitative shear wave ultrasound elastography: initial experience in solid breast masses. Breast Cancer Res. 2010;12(6):R104. doi: 10.1186/bcr2787. [PubMed: 21122101].

17. Sebag F, Vaillant-Lombard J, Berbis J, Griset V, Henry JF, Petit P, et al. Shear wave elastography: a new ultrasound imaging mode for the differential diagnosis of benign and malignant thyroid nodules. J Clin Endocrinol Metab. 2010;95(12):5281-8. doi: 10.1210/jc.2010-0766. [PubMed: 20881263].

18. Lalitha P, Reddy M, Reddy KJ. Musculoskeletal applications of elastography: a pictorial essay of our initial experience. Korean J Radiol. 2011;12(3):365-75. doi:10.3348/kjr.2011.12.3.365. [PubMed: 21603296].

19. Zeiter JH, Shin DH, Juzych MS, Jarvi TS, Spoor TC, Zwas F. Visual field defects in patients with normal-tension glaucoma and patients with high-tension glaucoma. Am J Ophthalmol. 1992;114(6):758-63. [PubMed: 1463046].

20. Brusini P, Filacorda S. Enhanced Glaucoma Staging System (GSS 2) for classifying functional damage in glaucoma. J Glaucoma. 2006;15(1):40-6. [PubMed: 16378017].

21. Ren R, Wang N, Li B, Li L, Gao F, Xu X, et al. Lamina cribrosa and peripapillary sclera histomorphometry in normal and advanced glaucomatous Chinese eyes with various axial length. Invest Ophthalmol Vis Sci. 2009;50(5):2175-84. doi: 10.1167/iovs.07-1429. [PubMed: 19387083].

22. Yang H, Downs JC, Girkin C, Sakata L, Bellezza A, Thompson H, et al 3-D histomorphometry of the normal and early glaucomatous monkey optic nerve head: lamina cribrosa and peripapillary scleral position and thickness. Invest Ophthalmol Vis Sci. 2007;48(10):4597-607. doi: 10.1167/iovs.07-0349. [PubMed: 17898283].

23. Yang H, Downs JC, Bellezza A, Thompson H, Burgoyne CF. 3-D histomorphometry of the normal and early glaucomatous monkey optic nerve head: prelaminar neural tissues and cupping. Invest Ophthalmol Vis Sci. 2007;48(11):5068-84. doi: 10.1167/iovs.07-0790. [PubMed: 17962459].

24. Sigal IA, Flanagan JG, Tertinegg I, Ethier CR. Finite element modeling of optic nerve head biomechanics. Invest Ophthalmol Vis Sci. 2004;45(12):4378-87. doi: 10.1167/iovs.04-0133. [PubMed: 15557446].

25. Agladioglu K, Pekel G, Altintas Kasikci S, Yagci R, Kiroglu Y. An evaluation of ocular elasticity using real-time ultrasound elastography in primary open-angle glaucoma. Br J Radiol. 2016;89(1060):20150429. doi: 10.1259/bjr.20150429. [PubMed: 26838949]. 